- Original Article

\title{
The Risk of Microalbuminuria by Obesity Phenotypes according to Metabolic Health and Obesity: The Korean National Health and Nutrition Examination Survey 2011-2014
}

\author{
Inyoung Choi', Heesun Moon', So Young Kang', Hyeonyoung Ko', Jinyoung Shin², Jungkwon Lee ${ }^{1, *}$ \\ 'Department of Family Medicine, Samsung Medical Center, Sungkyunkwan University School of Medicine, Seoul, Korea \\ ${ }^{2}$ Department of Family Medicine, Konkuk University Medical Center, Konkuk University School of Medicine, Seoul, Korea
}

Background: The present study aimed at identifying the difference in the risk of microalbuminuria among individuals with various obesity phenotypes in terms of metabolic health and obesity.

Methods: This cross-sectional study included 15,268 individuals and used data from the National Health and Nutrition Survey conducted from 2011 to 2014 . Obesity was defined as body mass index $\geq 25 \mathrm{~kg} / \mathrm{m}^{2}$. Metabolically unhealthy was defined as meeting two or more of the following criteria: systolic and diastolic blood pressure $\geq 130 / 85$ $\mathrm{mm} \mathrm{Hg}$ or current use of hypertensive drugs; triglyceride level $\geq 150 \mathrm{mg} / \mathrm{dL}$; high-density lipoprotein level $<40 / 50$ $\mathrm{mg} / \mathrm{dL}$ (in both men and women); and fasting blood glucose level $\geq 100 \mathrm{mg} / \mathrm{dL}$ or current use of oral antidiabetic medications. The participants were further classified into four subgroups: metabolically healthy non-obese (MHNO), metabolically healthy obese (MHO), metabolically unhealthy non-obese (MUNO), and metabolically unhealthy obese (MUO).

Results: A significant difference was observed in the microalbuminuria ratio among the four groups. The MHNO group was considered as the reference group, and the MHO, MUNO, and MUO groups were at an increased risk for microalbuminuria by 1.42 fold (95\% confidence interval [95\% CI], 1.03-1.96), 2.02 fold (95\% CI, 1.61-2.53), and 3.40 fold (95\% CI, 2.70-4.26), respectively, after adjusting confounding factors.

Conclusion: The MUNO group had a higher risk of developing microalbuminuria than the MHNO group. Thus, based on this result, differences were observed in the risk of developing microalbuminuria among individuals with various obesity subtypes.

Keywords: Obesity; Metabolic Diseases; Albuminuria; Creatinine

Received: December 29, 2016, Revised: May 19, 2017, Accepted: May 23, 2017

*Corresponding Author: Jungkwon Lee https://orcid.org/0000-0001-5503-9605

Tel: +82-2-3410-2441, Fax: +82-2-3410-0088, E-mail: jklee@skku.edu 


\section{INTRODUCTION}

The incidence of obesity continues to rapidly increase worldwide, and this condition does not only have health-related but also social, psychological, and economic impacts. In Korea, the estimated socioeconomic cost, including direct and indirect costs, of obesity was approximately $\$ 1,787$ million ( $\$ 1,081$ million for men and $\$ 706$ million for women). These total costs account for about $0.22 \%$ of the gross domestic product and $3.7 \%$ of the national health care expenditures in 2005. ${ }^{1)}$ Moreover, obesity increases the risk for cardiovascular diseases and mortality ${ }^{2}$ and is known as a risk factor for kidney diseases. ${ }^{3)}$ Metabolic disorder affects kidney function and is a well-known risk factor for cardiovascular morbidity and mortality. ${ }^{4,5)}$ Although it is assumed that the presence of metabolic syndrome may influence the effect of obesity on cardiovascular and kidney functions, there is no consensus on this subject. ${ }^{6-10)}$ In particular, whether metabolically healthy obese (MHO) individuals have a better prognosis remains controversial. ${ }^{10,11}$

The pathophysiology of obesity and metabolic disorder can be partly explained by microvascular dysfunction. ${ }^{12,13)}$ Microalbuminuria is a well-known marker of microvascular dysfunction. ${ }^{14)}$ This condition is defined as the excretion of albumin in the urine at $30-300 \mathrm{mg} / \mathrm{d}$. It reflects early kidney injury and is associated with an increased risk for cardiovascular disease and mortality. ${ }^{15)}$ In addition, a study on patients with cardiovascular or cerebrovascular diseases has reported the association between microalbuminuria and increased mortality, even though the cutoff value for microalbuminuria was lower than the normal value. ${ }^{16)}$ Previous studies on the association between obesity and microalbuminuria have been conducted. ${ }^{17,18)}$ However, the correlation between metabolic syndrome and microalbuminuria has not been fully elucidated. ${ }^{19-21)}$ Therefore, this study aimed at investigating the differences in the risk for microalbuminuria among individuals with various obesity phenotypes according to the presence or absence of metabolic disorders.

\section{METHODS}

\section{Study Population}

The Korea National Health and Nutrition Examination Survey (KNHANES) is a series of national surveys on the health and nutritional status of individuals in Korean, and it is in accordance with Article 16 of the National Health Promotion Act. It was first conducted in 1998 and then in 2002, 2005, 2007-2009, 2010-2012, and 2013-2015. The KNHANES randomly assesses 10,000 individuals per year. The sample design was stratified according to city and province, housing type, and intrinsic floor based on stratified colony extraction. The households were selected via systematic sampling, and a two-step sampling was performed. $^{22)}$

In this study, data obtained from the KNHANES (2011-2014) were used. Among the 23,688 adults aged 19 years and older, 15,541 were included in the study after excluding patients with an albumin-creatinine ratio of more than $300 \mathrm{mg} / \mathrm{g}$, a fasting duration less than 12 hours, or an estimated glomerular filtration rate below $60 \mathrm{~mL} / \mathrm{min} / 1.73 \mathrm{~m}^{2}$. In addition, a total of 15,268 patients were finally included after excluding 273 patients with missing data on body mass index (BMI) or the adult treatment panel (ATP)-III metabolic syndrome criteria. The study protocol was approved by the Institutional Review Board of Samsung Medical Center (IRB approval no., SMC 2016-08-182).

\section{Data Collection and Measurement of Factors}

The data on socioeconomic variables collected via interviews during the KNAHNES included sex, age, education level, and income, and the health-related behavioral variables, such as smoking, drinking, exercise, results of the food intake survey, and use of medications, and were collected via self-completed questionnaires. Education level was classified into four groups: elementary school graduate or lower, middle school graduate, high school graduate, and college graduate or higher. The income level was classified into four categories by dividing the monthly average household income into four quartiles. Daily energy intake was calculated based on the results of the food intake survey, including food type and frequency. Height, weight, and waist circumference were measured, and BMI was calculated based on height and weight. Blood pressure was measured for three consecutive times using a mercury sphygmomanometer (Baumanometer Wall Unit 33; WA Baum Co. Inc., Copiagne, NY, USA). The averages of the systolic and diastolic blood pressures were obtained using the first and second measurements. After fasting for more than 12 hours and excluding patients with an estimated glomerular filtration rate below $60 \mathrm{~mL} /$ $\mathrm{min} / 1.73 \mathrm{~m}^{2}$, fasting glucose, glycated hemoglobin, total cholesterol, triglyceride, high-density lipoprotein (HDL), aspartate transaminase (AST), alanine transaminase (ALT), and serum creatinine levels were automatically measured using a Hitachi Automatic Analyzer 7600 (Hitachi, Tokyo, Japan). Random urinalysis was performed to measure the levels of albumin (turbidimetric immunoassay; Hitachi Automatic Analyzer 7600) and creatinine (colorimetric method; Hitachi Automatic Analyzer 7600) excreted in the urine.

\section{Definition of the Variables}

Obesity was defined as BMI $\geq 25 \mathrm{~kg} / \mathrm{m}^{2}$ based on the Asia-Pacific guidelines of the World Health Organization. ${ }^{23)}$ The metabolically unhealthy group included patients who met at least two of the following criteria according to the National Cholesterol Education Program ATPIII guidelines for metabolic syndrome, ${ }^{24)}$ except for waist circumference: ${ }^{10,11,25)}(1)$ systolic and diastolic blood pressure $\geq 130 / 85 \mathrm{~mm} \mathrm{Hg}$ or the current use of hypertensive drugs, (2) triglyceride level $\geq 150 \mathrm{mg} /$ $\mathrm{dL}$, (3) HDL level $<40 / 50 \mathrm{mg} / \mathrm{dL}$ (in both men and women), and (4) fasting blood glucose level $\geq 100 \mathrm{mg} / \mathrm{dL}$ or the current use of oral antidiabetic medications. The participants were further classified into four subgroups according to the presence of obesity and/or metabolic disorders: (1) metabolically healthy non-obese (MHNO), (2) MHO, (3) metabolically unhealthy non-obese (MUNO), and (4) metabolically unhealthy obese (MUO). Microalbuminuria was defined as an albumin-creatinine ratio of $30-300 \mathrm{mg} / \mathrm{g}$ according to the definition of the 
National Kidney Foundation. ${ }^{26)}$

Smokers were classified as smokers or non-smokers based on their current smoking status. Drinking was defined as regular alcohol consumption of more than $40 \mathrm{~g}$ per day in men and $20 \mathrm{~g}$ per day in women. ${ }^{27)}$ Physical activity was defined as high-intensity exercise more than 3 times a week for 20 minutes or moderate-intensity exercise 5 times a week for more than 30 minutes. $^{28)}$

\section{Statistical Analysis}

Collected data were analyzed using IBM SPSS ver. 22.0 (IBM Corp., Armonk, NY, USA). A P-value $<0.05$ was considered statistically significant. In Table 1, P-values were obtained using analysis of variance or chi-square test. Logistic regression analysis was carried out to calculate odds ratio (OR) and 95\% confidence intervals (95\% CIs), thus identify- ing the differences in the risk of microalbuminuria according to BMI, ATP-III components, and metabolically healthy state, as shown in Table 2 . Table 3 also shows the results of the logistic regression analysis on the differences in the risk for microalbuminuria in the subgroups according to obesity phenotype. Models 1 and 2 were obtained via univariate and multivariate analyses, respectively, after adjusting for covariates, such as sex and age, and model 3 was obtained via multivariate analysis after adjusting for sex, age, smoking status, alcohol consumption, physical activity, AST and ALT levels, education level, economic status, and daily energy intake.

In the KNHANES, when weights are used in data analysis, inclusion errors, unequal extraction rates, and the non-responding errors of the respondents are corrected. Therefore, the representativeness and accuracy of the target population, the health behavior of individuals in

Table 1. Characteristics of the subgroups with different obesity phenotypes $(N=15,268)$

\begin{tabular}{|c|c|c|c|c|c|}
\hline Characteristic & MHNO $(n=7,016)$ & $\mathrm{MHO}(\mathrm{n}=1,936)$ & MUNO $(n=3,350)$ & $\operatorname{MUO}(n=2,966)$ & P-value* \\
\hline Sex & & & & & $<0.01$ \\
\hline Men & 45.0 & 53.1 & 51.8 & 58.8 & \\
\hline Women & 55.0 & 46.9 & 48.2 & 41.2 & \\
\hline Age $(y)$ & $41.43 \pm 0.24$ & $41.98 \pm 0.410$ & $55.49 \pm 0.34$ & $50.58 \pm 0.37$ & $<0.01$ \\
\hline Height (cm) & $164.45 \pm 0.14$ & $165.26 \pm 0.28$ & $162.31 \pm 0.21$ & $164.49 \pm 0.23$ & $<0.01$ \\
\hline Waist circumference (cm) & $74.87 \pm 0.12$ & $88.80 \pm 0.23$ & $80.46 \pm 0.16$ & $91.77 \pm 0.19$ & $<0.01$ \\
\hline Body mass index ${ }^{\dagger}\left(\mathrm{kg} / \mathrm{m}^{2}\right)$ & $21.59 \pm 0.03$ & $27.32 \pm 0.07$ & $22.74 \pm 0.04$ & $27.89 \pm 0.07$ & $<0.01$ \\
\hline Systolic blood pressure (mm Hg) & $110.40 \pm 0.20$ & $115.58 \pm 0.37$ & $125.92 \pm 0.42$ & $126.59 \pm 0.36$ & $<0.01$ \\
\hline Diastolic blood pressure (mm Hg) & $72.16 \pm 0.16$ & $75.73 \pm 0.29$ & $79.09 \pm 0.25$ & $82.07 \pm 0.24$ & $<0.01$ \\
\hline Fasting plasma glucose level (mg/dL) & $90.25 \pm 0.18$ & $92.74 \pm 0.32$ & $106.33 \pm 0.56$ & $108.71 \pm 0.56$ & $<0.01$ \\
\hline Total cholesterol level (mg/dL) & $183.08 \pm 0.52$ & $194.34 \pm 0.96$ & $193.26 \pm 0.85$ & $199.48 \pm 0.90$ & $<0.01$ \\
\hline Triglyceride level (mg/dL) & $89.26 \pm 0.79$ & $110.59 \pm 1.87$ & $184.62 \pm 2.75$ & $210.37 \pm 3.30$ & $<0.01$ \\
\hline High-density lipoprotein cholesterol level (mg/dL) & $55.71 \pm 0.18$ & $51.63 \pm 0.28$ & $45.06 \pm 0.23$ & $43.35 \pm 0.21$ & $<0.01$ \\
\hline Alanine aminotransferase level (IU/L) & $20.14 \pm 0.19$ & $22.32 \pm 0.27$ & $23.76 \pm 0.31$ & $26.23 \pm 0.33$ & $<0.01$ \\
\hline Aspartate aminotransferase level (IU/L) & $17.52 \pm 0.29$ & $25.46 \pm 0.56$ & $22.78 \pm 0.36$ & $32.42 \pm 0.82$ & $<0.01$ \\
\hline Smoking status & & & & & $<0.01$ \\
\hline Current smoker & 21.5 & 25.1 & 26.9 & 28.1 & \\
\hline Current non-smoker & 78.5 & 74.9 & 73.1 & 71.9 & \\
\hline \multicolumn{6}{|l|}{ Drinking status ${ }^{\ddagger}$} \\
\hline Regular drinker & 3.3 & 4.6 & 6.0 & 6.8 & $<0.01$ \\
\hline Non-heavy drinker & 96.7 & 95.4 & 94.0 & 93.2 & \\
\hline Physical activity ${ }^{\S}$ & 40.5 & 44.5 & 34.2 & 38.4 & $<0.01$ \\
\hline \multicolumn{6}{|l|}{ Years of education (y) } \\
\hline$\leq 6$ & 10.4 & 12.6 & 32.4 & 24.5 & $<0.01$ \\
\hline $6-9$ & 7.2 & 8.4 & 14.2 & 13.1 & \\
\hline $10-12$ & 42.9 & 43.3 & 30.5 & 35.8 & \\
\hline$\geq 13$ & 39.5 & 35.8 & 22.9 & 26.7 & \\
\hline \multicolumn{6}{|l|}{ Economic status" } \\
\hline Low & 11.0 & 12.4 & 22.8 & 17.3 & $<0.01$ \\
\hline Moderately low & 26.5 & 27.5 & 26.4 & 26.6 & \\
\hline Moderately high & 30.1 & 32.1 & 25.2 & 30.0 & \\
\hline High & 32.5 & 27.9 & 25.6 & 26.0 & \\
\hline Daily energy intake (kcal) & $2,057.92 \pm 14.21$ & $2,148.63 \pm 35.38$ & $1,958.07 \pm 21.56$ & $2,129.82 \pm 27.39$ & $<0.01$ \\
\hline Albumin-creatinine ratio (mg/g) & $6.11 \pm 0.21$ & $7.09 \pm 0.41$ & $13.16 \pm 0.62$ & $14.74 \pm 0.63$ & $<0.01$ \\
\hline
\end{tabular}

Values are presented as \% or mean \pm standard deviation, unless otherwise stated.

MHNO, metabolically healthy non-obese; MHO, metabolically healthy obese; MUNO, metabolically unhealthy non-obese; MUO, metabolically unhealthy obese.

${ }^{*}$ Obtained using analysis of variance with a weighted composite sample. ${ }^{\dagger}$ Calculated as weight in kilograms divided by height in meters squared. ${ }^{\star}$ Regular drinkers include women drinking more than $20 \mathrm{~g}$ of alcohol per day and men more than $40 \mathrm{~g}$ per day. ${ }^{\S} \mathrm{A}$ history of high-intensity exercise at least 3 times in a week, which includes running, hiking, and fast cycling for more than 20 minutes at a time, or moderate-intensity exercise at least 5 times in a week, which include slow swimming, tennis (doubles), and volleyball for more than 30 minutes at a time. "Four levels of income quartile. 
Table 2. Odds ratios for microalbuminuria according to BMI, ATP-III components, and metabolically healthy state

\begin{tabular}{lllc}
\hline \multicolumn{1}{c}{ Variable } & Model 1 & Model 2 & Model 3 \\
\hline BMl & & & \\
$\quad$ Non-obese & 1.00 (Ref) & 1.00 (Ref) & 1.000 (Ref) \\
$\quad$ Obese & $1.92(1.64-2.26)$ & $1.89(1.61-2.23)$ & $1.90(1.59-2.28)$ \\
ATP-III components & & & \\
$\quad$ Blood pressure $\geq 130 / 85 \mathrm{~mm} \mathrm{Hg}$ or the use of antihypertensive drugs & $2.97(2.48-3.56)$ & $2.43(2.02-2.92)$ & $2.33(1.91-2.85)$ \\
$\quad$ Triglyceride level $\geq 150 \mathrm{mg} / \mathrm{dL}$ & $1.36(1.16-1.60)$ & $1.55(1.32-1.82)$ & $1.39(1.17-1.66)$ \\
$\quad$ High-density lipoprotein cholesterol level $\leq 50 \mathrm{mg} / \mathrm{dL}$ ( $\leq 40 \mathrm{mg} / \mathrm{dL}$ for women) & $1.19(1.01-1.39)$ & $0.96(0.81-1.15)$ & $0.97(0.81-1.16)$ \\
$\quad$ Fasting plasma glucose level $\geq 100 \mathrm{mg} / \mathrm{dL}$ or the use of oral antidiabetic drugs & $2.02(1.69-2.41)$ & $1.82(1.52-2.18)$ & $1.84(1.52-2.24)$ \\
$\quad$ Metabolic health & & & \\
$\quad$ Metabolically healthy & 1.00 (Ref) & 1.00 (Ref) & 1.00 (Ref) \\
$\quad$ Metabolically unhealthy & 3.49 (2.97-4.09) & $2.59(2.18-3.08)$ & $2.44(2.02-2.95)$ \\
\hline
\end{tabular}

Values are presented as OR $(95 \% \mathrm{Cl})$. OR and $95 \% \mathrm{Cl}$ were calculated via logistic regression analysis using a weighted composite sample. Model 1 was unadjusted. Model 2 was adjusted for age and sex. Model 3 was adjusted for the variables in model 2 plus smoking status, alcohol consumption, physical activity, alanine aminotransferase and aspartate aminotransferase level, years of education, economic status, and daily energy intake.

BMI, body mass index; ATP-III, adult treatment panel-III; Ref, reference; OR, odds ratio; Cl, confidence interval.

Table 3. Logistic regression analysis of the association between different obesity phenotypes and microalbuminuria

\begin{tabular}{lcccc}
\hline \multicolumn{1}{c}{ Variable } & No. of cases (\%) & Model 1 & Model 2 & Model 3 \\
\hline Metabolically healthy non-obese & $7,793(17.0)$ & 1 (Ref) & 1 (Ref) & 1 (Ref) \\
Metabolically healthy obese & $3,026(15.6)$ & $1.41(1.06-1.88)$ & $1.41(1.05-1.88)$ & $1.49(1.03-1.96)$ \\
Metabolically unhealthy non-obese & $2,402(16.7)$ & $3.19(2.61-3.90)$ & $2.16(1.74-2.67)$ & $2.02(1.61-2.53)$ \\
Metabolically unhealthy obese & $2,794(50.6)$ & $4.44(3.64-5.42)$ & $3.53(2.88-4.34)$ & $3.40(2.70-4.26)$
\end{tabular}

Values are presented as OR (95\% Cl). OR and $95 \% \mathrm{Cl}$ were calculated via logistic regression analysis using a weighted composite sample. Model 1 was unadjusted. Model 2 was adjusted for age and sex. Model 3 was adjusted for the variables in model 2 plus smoking status, alcohol consumption, physical activity, alanine aminotransferase and aspartate aminotransferase level, years of education, economic status, and daily energy intake.

$\mathrm{OR}$, odds ratio; $\mathrm{Cl}$, confidence interval; Ref, reference.

Korea, chronic diseases, and food and nutrient intake may be modified. In this study, all statistical analyses were performed using a weighted composite sample.

\section{RESULTS}

For each obesity subtype group, a total of 7,016 (49.7\%), 1,936 (14.5\%), 3,350 (19.7\%), and 2,966 (19.4\%) participants were included in the MHNO, MHO, MUNO, and MUO groups, respectively. The baseline characteristics of the respective groups are shown in Table 1. Significant differences were observed in all items among the groups. Microalbuminuria was observed in 1,003 (5.7\%) participants aged $\geq 19$ years.

As shown in Table 2 , obesity (BMI $\geq 25 \mathrm{~kg} / \mathrm{m}^{2}$ ) was associated with an increased risk of microalbuminuria in every model, with ORs of 1.92 (95\% CI, 1.64-2.26), 1.89 (95\% CI, 1.61-2.23), and 1.90 (95\% CI, 1.59-2.28) for models 1, 2, and 3, respectively. Every ATP-III factor, except abdominal obesity, was significantly associated with an increased risk of developing microalbuminuria in model 1. After adjusting for age, sex, smoking status, alcohol drinking status, physical activity, AST and ALT levels, education level, economic status, and daily energy intake, high blood pressure (OR, 2.33; 95\% CI, 1.91-2.85), high glucose level (OR, 1.84; 95\% CI, 1.52-2.24), and high triglyceride level (OR, 1.39; 95\% CI, 1.17-1.66) were significantly correlated with microalbuminuria in model 3 . The participants in the metabolically unhealthy groups, defined as having two or more ATP-III factors other than abdominal obesity, were 2.44 (95\% CI, 2.02-2.95) times more at risk of developing microalbuminuria than those in the metabolically healthy group.

Comparison results of the incidence of microalbuminuria among all four groups are shown in Table 3. Differences in the ratio of microalbuminuria among individuals with different subtypes were confirmed in all models. The MHNO group was considered as the reference group. The MHO, MUNO, and MUO groups were at an increased risk of developing microalbuminuria by 1.42 fold (95\% CI, 1.03-1.96), 2.02 fold (95\% CI, 1.61-2.53), and 3.40 fold (95\% CI, 2.70-4.26), respectively, after adjusting for confounding factors.

\section{DISCUSSION}

The results of the present study showed that the MUO group was at the highest risk of developing microalbuminuria, followed by the MUNO and MHO groups. In particular, the MUNO group was significantly at risk for microalbuminuria compared to the MHNO group. In other words, even in the groups with normal BMI, metabolically unhealthy participants were at an increased risk of developing microalbuminuria than the metabolically healthy participants. This finding suggests that metabolic disorder is associated with the development of microalbuminuria, which is consistent with the findings of previous studies that 
emphasized the importance of metabolic health. ${ }^{6,9,10)}$

Hamer and Stamatakis ${ }^{6}$ have reported that the MHO group was not at a higher risk for cardiovascular diseases or death compared with the MHNO group, and if an individual is diagnosed with a metabolic disorder, the risk for cardiovascular diseases and mortality increases regardless of the presence of obesity. In contrast, Hinnouho et al. ${ }^{10,11)}$ have concluded that the obese group was at a similar risk for cardiovascular diseases and death regardless of metabolic health, and the MUO group was at a higher risk for type 2 diabetes mellitus. Calori et al. ${ }^{9)}$ have reported that obesity along with insulin resistance was not associated with an overall increased risk for cancer- or cardiovascular disease-related death unlike obesity alone. In Korea, a 10.3-year follow-up study on 2,317 elderly individuals aged $\geq 60$ years showed that the MUNO group was at a higher risk for mortality than the MHO group. $^{29)}$

The results of the present study showed a higher prevalence of microalbuminuria in the MHO group than in the MHNO group. This finding supports the results of a previous meta-analysis showing that the obese groups were at a higher risk for cardiovascular diseases and cardiovascular disease-related death and higher carotid intimal thickness than the non-obese groups ${ }^{30)}$ A previous study has confirmed the correlation between obesity and microalbuminuria, ${ }^{17)}$ although some studies have claimed that this result was not valid or only applicable in certain subgroups. ${ }^{19-21)}$ In a study on Korean participants, obesity was independently associated with microalbuminuria, which is similar to other factors related to metabolic syndrome. ${ }^{29)}$

The definition of metabolic disorder varies among studies but generally involves the criteria for metabolic syndrome or surrogate markers of insulin resistance. ${ }^{30)}$ Because the markers for insulin resistance could not be acquired owing to the limitations of the KNHANES data, metabolic disorder was defined as the presence of two or more of the criteria (excluding waist circumference) for metabolic syndrome. This was consistent with the results of previous studies by Ortega et al. ${ }^{25}$ and Hinnouho et al. ${ }^{11,12)}$ The waist circumference criterion was excluded because it has collinearity with BMI, as also shown in previous studies. $^{10,11,25)}$

The present study has a key strength in that the use of the KNHANES data made the results generalizable to Korean adults partly due to the large sample size. However, the causal relationship could not be clarified, which a well-known limitation of cross-sectional studies and could not be avoided. Second, microalbuminuria was assessed only once. Thus, there was a possibility of error. Furthermore, data on the use of concomitant medications, including antihypertensive, antidiabetic, and lipid-lowering drugs, were included. However, data on the components of each drug were not considered, which another limitation of this study. Therefore, patients who were taking antihypertensive and antidiabetic drugs were considered to have high blood pressure and high fasting blood glucose levels, respectively. However, those who were taking medications for dyslipidemia were not considered to have high triglyceride or low HDL-cholesterol levels.

In conclusion, this cross-sectional study that targeted Korean adults confirmed differences in the risk for microalbuminuria among individuals with various obesity phenotypes. The MUNO group was at a higher risk for microalbuminuria than the MHNO group, suggesting that the prevention of several chronic diseases is necessary if the signs of metabolic disorder are evident regardless of normal body weight.

\section{CONFLICT OF INTEREST}

No potential conflict of interest relevant to this article was reported.

\section{ACKNOWLEDGMENTS}

We would like to thank the Korea Centers for Disease Control and Prevention for the data obtained from the Fifth and Sixth Korea National Health and Nutrition Examination Survey (KNHANES V \& VI, 20112014).

\section{REFERENCES}

1. Kang JH, Jeong BG, Cho YG, Song HR, Kim KA. Socioeconomic costs of overweight and obesity in Korean adults. J Korean Med Sci 2011;26:1533-40.

2. Zhang C, Rexrode KM, van Dam RM, Li TY, Hu FB. Abdominal obesity and the risk of all-cause, cardiovascular, and cancer mortality: sixteen years of follow-up in US women. Circulation 2008;117:1658-67.

3. Wang Y, Chen X, Song Y, Caballero B, Cheskin LJ. Association between obesity and kidney disease: a systematic review and meta-analysis. Kidney Int 2008;73:19-33.

4. Galassi A, Reynolds K, He J. Metabolic syndrome and risk of cardiovascular disease: a meta-analysis. Am J Med 2006;119:812-9.

5. Thomas G, Sehgal AR, Kashyap SR, Srinivas TR, Kirwan JP, Navaneethan SD. Metabolic syndrome and kidney disease: a systematic review and meta-analysis. Clin J Am Soc Nephrol 2011;6:2364-73.

6. Hamer M, Stamatakis E. Metabolically healthy obesity and risk of allcause and cardiovascular disease mortality. J Clin Endocrinol Metab 2012;97:2482-8.

7. Appleton SL, Seaborn CJ, Visvanathan R, Hill CL, Gill TK, Taylor AW, et al. Diabetes and cardiovascular disease outcomes in the metabolically healthy obese phenotype: a cohort study. Diabetes Care 2013;36:2388-94.

8. Kuk JL, Ardern CI. Are metabolically normal but obese individuals at lower risk for all-cause mortality? Diabetes Care 2009;32:2297-9.

9. Calori G, Lattuada G, Piemonti L, Garancini MP, Ragogna F, Villa M, et al. Prevalence, metabolic features, and prognosis of metabolically healthy obese Italian individuals: the Cremona Study. Diabetes Care 2011;34:210-5.

10. Hinnouho GM, Czernichow S, Dugravot A, Nabi H, Brunner EJ, Kivimaki M, et al. Metabolically healthy obesity and the risk of cardiovascular disease and type 2 diabetes: the Whitehall II cohort study. Eur Heart J 2015;36:551-9.

11. Hinnouho GM, Czernichow S, Dugravot A, Batty GD, Kivimaki M, Singh-Manoux A. Metabolically healthy obesity and risk of mortality: does the definition of metabolic health matter? Diabetes Care 2013;36: 2294-300. 
12. Serne EH, de Jongh RT, Eringa EC, IJzerman RG, Stehouwer CD. Microvascular dysfunction: a potential pathophysiological role in the metabolic syndrome. Hypertension 2007;50:204-11.

13. Czernichow S, Greenfield JR, Galan P, Bastard JP, Charnaux N, Samaras $\mathrm{K}$, et al. Microvascular dysfunction in healthy insulin-sensitive overweight individuals. J Hypertens 2010;28:325-32.

14. Futrakul N, Sridama V, Futrakul P. Microalbuminuria: a biomarker of renal microvascular disease. Ren Fail 2009;31:140-3.

15. Chronic Kidney Disease Prognosis Consortium, Matsushita K, van der Velde M, Astor BC, Woodward M, Levey AS, et al. Association of estimated glomerular filtration rate and albuminuria with all-cause and cardiovascular mortality in general population cohorts: a collaborative meta-analysis. Lancet 2010;375:2073-81.

16. Klausen KP, Scharling H, Jensen JS. Very low level of microalbuminuria is associated with increased risk of death in subjects with cardiovascular or cerebrovascular diseases. J Intern Med 2006;260:231-7.

17. Chang A, van Horn L, Jacobs DR Jr, Liu K, Muntner P, Newsome B, et al. Lifestyle-related factors, obesity, and incident microalbuminuria: the CARDIA (Coronary Artery Risk Development in Young Adults) study. Am J Kidney Dis 2013;62:267-75.

18. Kim H, Kim HJ, Shin N, Han M, Park H, Kim M, et al. Visceral obesity is associated with microalbuminuria in nondiabetic Asians. Hypertens Res 2014;37:679-84.

19. Rowley KG, Iser DM, Best JD, O’Dea K, Leonard D, McDermott R. Albuminuria in Australian Aboriginal people: prevalence and associations with components of the metabolic syndrome. Diabetologia 2000;43:1397-403.

20. Konta T, Hao Z, Abiko H, Ishikawa M, Takahashi T, Ikeda A, et al. Prevalence and risk factor analysis of microalbuminuria in Japanese general population: the Takahata study. Kidney Int 2006;70:751-6.

21. Lin CC, Liu CS, Li TC, Chen CC, Li CI, Lin WY. Microalbuminuria and the metabolic syndrome and its components in the Chinese popula- tion. Eur J Clin Invest 2007;37:783-90.

22. Kweon S, Kim Y, Jang MJ, Kim Y, Kim K, Choi S, et al. Data resource profile: the Korea National Health and Nutrition Examination Survey (KNHANES). Int J Epidemiol 2014;43:69-77.

23. WHO Expert Consultation. Appropriate body-mass index for Asian populations and its implications for policy and intervention strategies. Lancet 2004;363:157-63.

24. Expert Panel on Detection, Evaluation, and Treatment of High Blood Cholesterol in Adults. Executive summary of the third report of the National Cholesterol Education Program (NCEP) Expert Panel on Detection, Evaluation, and Treatment of High Blood Cholesterol in Adults (Adult Treatment Panel III). JAMA 2001;285:2486-97.

25. Ortega FB, Lee DC, Katzmarzyk PT, Ruiz JR, Sui X, Church TS, et al. The intriguing metabolically healthy but obese phenotype: cardiovascular prognosis and role of fitness. Eur Heart J 2013;34:389-97.

26. National Kidney Foundation. K/DOQI clinical practice guidelines for chronic kidney disease: evaluation, classification, and stratification. Am J Kidney Dis 2002;39(2 Suppl 1):S1-266.

27. Bardach AE, Caporale JE, Rubinstein AL, Danaei G. Impact of level and patterns of alcohol drinking on coronary heart disease and stroke burden in Argentina. PLoS One 2017;12:e0173704.

28. American College of Sports Medicine. ACSM's guidelines for exercise testing and prescription. 9th ed. Philadelphia (PA): Lippincott Williams \& Wilkins; 2013.

29. Choi KM, Cho HJ, Choi HY, Yang SJ, Yoo HJ, Seo JA, et al. Higher mortality in metabolically obese normal-weight people than in metabolically healthy obese subjects in elderly Koreans. Clin Endocrinol (Oxf) 2013;79:364-70.

30. Roberson LL, Aneni EC, Maziak W, Agatston A, Feldman T, Rouseff M, et al. Beyond BMI: the "metabolically healthy obese" phenotype \& its association with clinical/subclinical cardiovascular disease and allcause mortality: a systematic review. BMC Public Health 2014;14:14. 